\title{
NOUVELLE
}

\section{Adhérer ou ne pas adhérer}

\section{Stratégie adaptative de Plasmodium falciparum pour persister en saison sèche}

Catherine Bourgouin, Nicolas Puchot, Richard Paul

Unité de Génétique fonctionnelle des maladies infectieuses, Institut Pasteur, CNRS UMR 2000, 75015 Paris, France. catherine.bourgouin@pasteur.fr richard.paul@pasteur.fr
> Comment les populations de Plasmodium falciparum, un des parasites unicellulaires responsables du paludisme, peuvent-elles rester endémiques dans les régions du monde où la saison sèche et l'absence concomitante de moustiques vecteurs peuvent être très longues? Plusieurs hypothèses complémentaires, impliquant différents rôles des moustiques et des hommes, ont été avancées pour tenter de répondre à cette question d'un grand intérêt, notamment pour l'élimination du paludisme. Des travaux récents ont montré que les moustiques peuvent migrer, par vent fort, à partir de zones de transmission pérenne, et ainsi potentiellement importer des parasites avant l'expansion locale de la population de moustiques au début de la saison des pluies $[1,2](\rightarrow)$.

$\rightarrow$ Voir la Nouvelle de C. Bourgouin et R. Paul, $\mathrm{m} / \mathrm{s}$ $n^{\circ} 1$, janvier 2021, page 11

De plus, la présence de moustiques estivants (et potentiellement infectieux) a également été suggérée, fournissant ainsi un réservoir infectieux [3]. Cependant, malgré le rôle des moustiques estivants ou migrants, il est établi que les parasites persistent chez l'homme pendant de longues périodes sous forme d'infections chroniques et sont infectieux pour les moustiques [4]. L'homme constitue donc le réservoir de parasites sans doute le plus important. La question n'est donc pas de savoir si les parasites peuvent survivre, mais comment ils survivent sans stimuler trop fortement le système immunitaire, pendant une période où la transmission n'est pas possible en raison de l'absence des moustiques. Quel mécanisme permet aux parasites de persister à faible densité dans l'organisme sous la forme d'une infection chronique?

L'issue d'une infection par $P$. falciparum dépend beaucoup de la durée de l'exposition antérieure à ce parasite et du nombre des infections précédentes, donc du niveau d'endémicité. Le dogme est que le risque de développer un accès infectieux cliniquement apparent dépend de l'âge de l'individu et de l'intensité de la transmission : les premières infections entraînent une forte réponse fébrile, mais lors d'infections répétées, l'immunité clinique se développe, et les individus peuvent ensuite être infectés et rester asymptomatiques malgré une forte densité de parasites. L'immunité antiparasitaire se développe progressivement, et bien que les densités parasitaires diminuent avec l'âge et les antécédents d'exposition à l'infection au cours de la vie, cette immunité ne devient jamais stérilisante. Une deuxième caractéristique de ce parasite est que sa présence est associée à l'expression de molécules de cytoadhérence à la surface du globule rouge infecté, permettant la liaison de ce dernier aux cellules endothéliales de l'hôte et éviter ainsi sa destruction par la rate (Figure 1) [5]. En effet, la rate est responsable de l'élimination des globules rouges vieillissants, et l'infection de ces cellules par un parasite entraîne leur vieillissement prématuré. Parmi les molécules de cytoadhérence du parasite, les mieux décrites sont les variant surface antigens (VSA) de la famille P. falciparum erythrocyte membrane protein 1
(PfEMP1), codés par les gènes var $[6,7]$. Cependant, les VSA sont fortement immunogènes, et à chaque génération asexuée (toutes les 48 heures), un faible pourcentage de parasites exprime un nouveau VSA pour échapper à la réponse immunitaire dépendant des anticorps. Ainsi, les parasites ont développé ce mécanisme de cytoadhérence pour éviter la clairance splénique des globules rouges infectés, mais avec le risque de provoquer une réponse immunitaire humorale. Dans la mesure où il existe un nombre limité de VSA différents par génome parasitaire (environ 60), le répertoire complet de VSA devrait avoir été exprimé après quelques mois. Comment alors le parasite se maintient-il pendant la saison sèche, qui dure jusqu'à neuf mois dans certaines régions, sans recourir à une transmission par les moustiques vecteurs?

Andrade et al. répondent à cette question en explorant en détail les caractéristiques parasitaires et immunitaires des infections chroniques de la saison sèche par rapport à celles des infections cliniquement apparentes de la saison humide [8]. Dans une zone de transmission très saisonnière de la maladie au Mali, les auteurs ont suivi une cohorte d'environ 600 individus âgés de 3 mois à 45 ans pendant une durée de deux ans, couvrant les saisons pluvieuses (de juin à décembre) et sèches (de janvier à mai). La grande majorité des cas d'infection cliniquement apparente sont survenus pendant la saison des pluies de chaque année (386 et 347 cas, contre seulement 12 et 5 cas pendant la saison sèche, respectivement). En utilisant des données appariées du début (janvier) et 


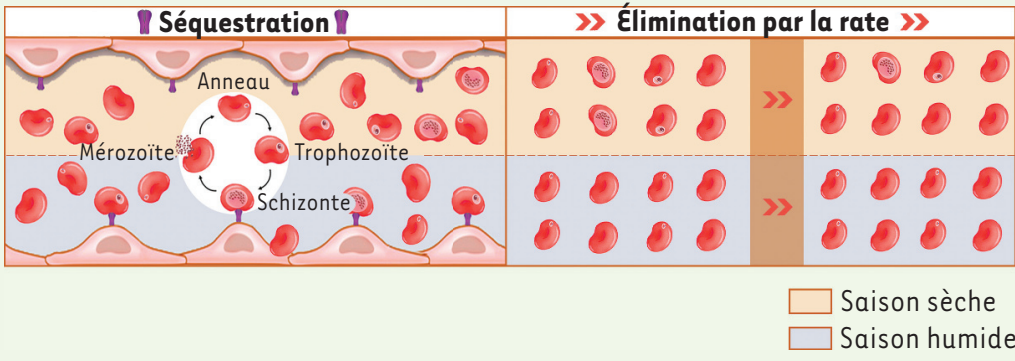

Figure 1. Représentation schématique de l'absence de séquestration, dans les vaisseaux sanguins, des globules rouges infectés par P. falciparum en saison sèche, et de leur rétention et destruction par la rate, par opposition à leur séquestration vasculaire, empêchant leur clairance splénique, lorsqu'ils sont infectés durant la saison humide. Un récepteur membranaire des cellules de l'endothélium vasculaire (représenté en violet) permet l'adhérence des globules rouges infectés par des parasites durant la saison humide, via notamment les ligands VSA (variant surface antigens) exprimés à la surface du globule rouge.

de la fin (mai) des saisons sèches pour plus de 400 individus, ils ont découvert, en utilisant la technique d'amplification de I'ADN par PCR (polymerase chain reaction) pour détecter le génome du parasite, que $20 \%$ des individus étaient en fait porteurs asymptomatiques de $P$. falciparum en janvier de chaque année, et que 12 à $15 \%$ de ces individus étaient toujours infectés en mai. Il est important de signaler qu'aucun individu non infecté au début de la saison sèche n'a été infecté pendant cette saison, ce qui confirme l'absence de transmission active du parasite pendant la saison sèche. Comme attendu avec le développement de l'immunité clinique en fonction de l'âge, les jeunes enfants étaient, à la fin de la saison sèche, porteurs asymptomatiques de $P$. falciparum moins fréquemment que les enfants plus âgés et les jeunes adultes. Les densités parasitaires étaient toutes très inférieures à celles des cas de paludisme cliniquement apparent observés au cours de la saison des pluies ou, beaucoup plus rarement, au cours de la saison sèche.

Les auteurs ont ensuite comparé, en janvier et en mai, le profil immunologique de porteurs asymptomatiques de parasites, âgés de 7 à 17 ans, avec celui des enfants et adolescents de même âge indemnes d'infection. II n'y avait pas de différences dans les marqueurs sérologiques inflammatoires, ni dans les taux de cytokines habituellement associées à l'infection parasitaire, ni dans les taux et proportions de cellules immunitaires (monocytes, lymphocytes $T$ et B). La seule différence entre ces deux groupes était une augmentation du nombre de lymphocytes $B$ mémoire ayant subi une commutation de classe (d'IgM à $I g G$ ), produisant des anticorps spécifiques anti-PfEMP, chez les individus porteurs du parasite. La capacité de réponse de ces lymphocytes anti-PfEMPl chez ces individus était plus élevée que chez les individus non porteurs, mais cette capacité avait diminué de janvier à mai, même chez les individus porteurs. De plus, bien que les taux d'anticorps contre les antigènes parasitaires «non VSA (en particulier contre ceux impliqués dans l'infection des globules rouges par le parasite) soient également plus élevés chez les porteurs de parasites, aucun effet neutralisant supérieur de leurs anticorps par rapport à ceux d'individus non porteurs n'a été observé sur l'infection des globules rouges par le parasite dans un test in vitro. Ainsi, l'infection infraclinique des individus par le parasite durant la saison sèche a apparemment très peu d'impact sur leur réponse immunitaire.

Les auteurs ont ensuite comparé le profil du transcriptome des parasites chez les porteurs asymptomatiques durant la saison sèche et chez les individus présentant une infection cliniquement apparente durant la saison des pluies. Ils ont observé des différences notables entre ces deux groupes pour de nombreux gènes (surexpression ou répression), en particulier ceux impliqués dans les voies métaboliques et dans la réplication ou la recombinaison homologue de I'ADN. Une analyse métabolomique détaillée du sérum des porteurs asymptomatiques durant la saison sèche et des individus présentant une infection symptomatique durant la saison des pluies n'a pas révélé d'impact des parasites sur la composition métabolique du sérum des porteurs de parasites quelle que soit la saison. Les auteurs se sont ensuite demandé si le taux de réplication des parasites au sein des globules rouges était modifié selon la saison, et étonnamment, ils ont constaté que les parasites collectés à la saison sèche se développaient apparemment plus rapidement in vitro que ceux collectés à la saison des pluies. Ils ont alors proposé une autre explication de cette observation: que les parasites présents dans le sang des porteurs durant la saison sèche soient simplement plus âgés que ceux normalement trouvés dans le sang des individus symptomatiques pendant la saison des pluies. Chez la plupart des individus infectés, l'expression de PfEMPl commence dans les parasites au stade «anneau», et la protéine est présente à la surface des globules rouges lorsque l'anneau mûrit en trophozoïte ${ }^{1}$ [7]. Ainsi, les trophozoïtes (et les schizontes $^{2}$ ) sont rarement observés dans le sang circulant, car ils sont séquestrés par cytoadhérence des globules rouges infectés aux cellules endothéliales vasculaires (Figure 1). En utilisant une estimation statistique pour déterminer l'âge des parasites en culture (défini comme le temps écoulé depuis l'entrée du parasite dans le globule rouge) sur la base de leur transcriptome, les auteurs ont noté que les profils transcriptomiques obtenus permettaient de distinguer les parasites

\footnotetext{
${ }^{1}$ Ce terme désigne la forme intraérythrocytaire du Plasmodium à son premier stade de développement.

2 Stade évolutif asexué des sporozoaires (Plasmodium en particulier), d'abord multinucléé, puis dont la division individualise les mérozoïtes qui infecteront d'autres hématies lorsqu'ils auront été libérés dans les sang par éclatement de I'hématie infectée.
} 


\section{GLOSSAIRE}

Accès palustre : état caractérisé par une fièvre supérieure à $37,5^{\circ} \mathrm{C}$ et par la présence d'au moins 2500 parasites par $\mu \mathrm{L}$ de sang au comptage par microscopie sur frottis sanguin.

Infection asymptomatique (ou infraclinique) : par opposition à l'accès palustre, état caractérisé par l'absence de fièvre associée et par un portage parasitaire très faible, souvent non détectable par microscopie et révélé par la technique PCR (polymerase chain reaction) d'amplification du génome parasitaire.

Cycle érythrocytaire de $\boldsymbol{P}$. falciparum : Anneau évoluant en trophozoïte, puis schizonte donnant naissance à de nombreux mérozoïtes ( 8 à 16) qui, après avoir été libérés dans la circulation sanguine, envahissent de nouveaux globules rouges (Figure 1 ).

Gamétocytes : forme sexuée du parasite ; ce sont les seuls à pouvoir se développer chez le moustique.

Microsphiltration : filtration à travers des microsphères métalliques de taille définie, imitant la filtration par la rate des globules rouges veillissants ou infectés.

circulants de la saison sèche (âgés d'environ 17 heures) des parasites circulants des sujets présentant une infection symptomatique (âgés d'environ 7 heures). Dans la mesure où les parasites plus âgés sont plus susceptibles d'être éliminés lors du passage dans la rate des globules rouges infectés, les auteurs ont utilisé la méthode de microsphiltration mécanique, qui imite la fonction de clairance de la rate [9], pour confirmer leur observation. En accord avec leur hypothèse, une proportion beaucoup plus importante de globules rouges infectés par les parasites de saison sèche était retenue par ce dispositif. Cependant, une nouvelle analyse du profil d'expression des gènes var à partir de l'analyse du transcriptome a révélé un enrichissement des transcrits var les plus exprimés dans les parasites issus des individus présentant une infection symptomatique par rapport aux parasites prélevés chez les individus porteurs durant la saison sèche, ce qui a conduit les auteurs à formuler l'hypothèse d'une cytoadhérence réduite des globules rouges infectés chez ces derniers (Figure 1). À l'aide de modèles mathématiques, les auteurs ont démontré que la réduction de la cytoadhérence des globules rouges infectés, avec leur élimination ultérieure par la rate, était suffisante pour expliquer les densités parasitaires réduites observées dans le portage asymptomatique de parasites en saison sèche.

Cette caractérisation par étapes des infections à $P$. falciparum en saison sèche fournit des preuves convaincantes de la modification du phénotype de cytoadhérence des globules rouges infectés par les parasites en saison sèche pour maintenir les densités parasitaires à de faibles niveaux. De ce fait, la réponse immunitaire pro-inflammatoire conduisant à des épisodes fébriles en raison de fortes densités de parasites n'est pas déclenchée, et la réponse immunitaire humorale avec stimulation des lymphocytes B mémoire, en particulier celles ciblant les antigènes associés à l'infection des globules rouges et à leur cytoadhérence à l'endothélium, est réduite au minimum. Ainsi, le parasite s'est apparemment adapté pour se maintenir à des niveaux très bas pendant la période sans pluie, où la transmission par les moustiques n'est pas possible. II a déjà été proposé que la très faible proportion des parasites qui modifient leur VSA à chaque génération s'explique par une exploitation prudente de l'hôte par le parasite. En effet, l'expression du même VSA devrait déclencher la réponse immunitaire de l'hôte et entraîner la mort du parasite (diminuant ainsi la charge parasitaire), mais on peut également considérer que la conservation du même VSA est un marqueur de «suicide», par lequel une partie de la population de parasites «se sacrifie» afin de prolonger l'infection sans tuer l'hôte [10]. II est à noter que deux autres espèces de para- sites du paludisme humain, $P$. vivax et $P$. malariae, n'envahissent que les globules rouges respectivement très jeunes ou âgés, limitant ainsi potentiellement leurs propres densités parasitaires [11].

II manque à cette étude approfondie sur le mécanisme de la survie de $P$. falciparum dans les globules rouges durant la saison sèche, la mesure de la production de gamétocytes, qui sont les stades parasitaires essentiels à la transmission aux moustiques. Bien qu'il soit démontré que des infections infracliniques chroniques chez l'homme sont infectieuses pour les moustiques [4], comprendre comment la transmission du parasite de l'homme vers le moustique est assurée dans ce contexte de densité parasitaire faible au début de la saison des pluies fournirait la dernière pièce du puzzle. On sait que les parasites du paludisme sont capables de répondre aux changements de leur environnement hématologique pour moduler la production de ces stades qui permettent la transmission aux moustiques vecteurs [12, 13]. Il a également été noté qu'au retour des moustiques, les densités de parasites asexués augmentent dans les infections chroniques [14]. Une telle augmentation de la densité parasitaire a été corrélée à une augmentation du nombre de piqûres de moustiques reçues, à la fois par l'étude d'une cohorte humaine et dans des modèles aviaires $[14,15]$. II est donc logique de supposer que le déclencheur de la diminution de la cytoadhérence des globules rouges infectés observée en saison sèche pourrait être l'absence de piqûres de moustiques [8]. Bien que P. falciparum soit un des agents pathogènes les plus étudiés, les stratégies d'adaptation que ce parasite unicellulaire utilise pour sa survie et sa transmission continuent d'étonner. $\diamond$

\section{To stick or not to stick: A Plasmodium falciparum adaptive strategy to persist during the dry season}

\section{LIENS D'INTÉRÊT}

Les auteurs déclarent n'avoir aucun lien d'intérêt concernant les données publiées dans cet article. 


\section{RÉFÉRENCES}

1. Huestis DL, Dao A, Diallo M, et al. Windborne long distance migration of malaria mosquitoes in the Sahel. Nature 2019; 574 : 404-8.

2. Bourgouin C, Paul R. Autant en emporte le vent: comment les anophèles recolonisent les régions arides du Sahel pour y transmettre le paludisme. Med Sci (Paris) $2021 ; 37: 11-4$

3. Omer SM, Cloudsley-Thompson JL. Dry season biology of Anopheles gambiae giles in the Sudan. Nature 1968 ; $217: 879-80$.

4. Barry A, Bradley J, Stone W, et al. Higher gametocyte production and mosquito infectivity in chronic compared to incident Plasmodium falciparum infections. Nat Commun $2021 ; 12: 2443$.

5. Chotivanich K, Udomsangpetch R, McGready R, et al. Central role of the spleen in malaria parasite clearance. J Infect Dis 2002 ; 185 : 1538-41.
6. Hviid L, Jensen ATR. PfEMPl, a parasite protein family of key importance in Plasmodium falciparum malaria immunity and pathogenesis. Adv Parasitol $2015 ; 88$ : 51-84.

7. Lee WC, Russell B, Rénia L. Sticking for a cause: The falciparum malaria parasites cytoadherence paradigm. Front Immunol $2019 ; 10: 1444$.

8. Andrade CM, Fleckenstein H, Thomson-Luque R, et al. Increased circulation time of Plasmodium falciparum underlies persistent asymptomatic infection in the dry season. Nat Med 2020 ; 26 : 1929-40.

9. Deplaine G, Safeukui I, Jeddi F, et al. The sensing of poorly deformable red blood cells by the human spleen can be mimicked in vitro. Blood $2011 ; 117$ : e88-95.

10. Saul $A$. The role of variant surface antigens on malaria infected red blood cells. Parasitol Today $1999 ; 15$ : 455-7.

11. Paul REL, Ariey F, Robert V. The evolutionary ecology of Plasmodium. Ecol Letters $2003 ; 6$ : 866-80.
12. Paul REL, Coulson TN, Raibaud A, Brey PT. Sex determination in malaria parasites. Science 2000 ; 287 : 128-31.

13. Brancucci NMB, Gerdt JP, Wang C, et al. Lysophosphatidylcholine regulates sexual stage differentiation in the human malaria parasite Plasmodium falciparum. Cell 2017 ; 171 : 1532-44.

14. Lawaly R, Konate L, Marrama L, et al. Impact of mosquito bites on asexual parasite density and gametocyte prevalence in asymptomatic chronic Plasmodium falciparum infections and correlation with Ig $\varepsilon$ and IgG titres. Infect Immun $2012 ; 80$ : 2240-6.

15. Cornet S, Nicot A, Rivero A, Gandon S. Evolution of plastic transmission strategies in avian malaria. PLoS Pathog 2014 ; 10 : el004308. 